\title{
Bifurcation Scenarios of Neural Firing Patterns across Two Separated Chaotic Regions as Indicated by Theoretical and Biological Experimental Models
}

\author{
Huaguang Gu, Baobao Pan, and Jian Xu \\ School of Aerospace Engineering and Applied Mechanics, Tongji University, Shanghai 200092, China \\ Correspondence should be addressed to Huaguang Gu; guhuaguang@tongji.edu.cn
}

Received 5 September 2013; Accepted 30 October 2013

Academic Editor: Massimiliano Ferrara

Copyright @ 2013 Huaguang Gu et al. This is an open access article distributed under the Creative Commons Attribution License, which permits unrestricted use, distribution, and reproduction in any medium, provided the original work is properly cited.

Nonlinear dynamics can be used to identify relationships between different firing patterns, which play important roles in the information processing. The present study provides novel biological experimental findings regarding complex bifurcation scenarios from period-1 bursting to period-1 spiking with chaotic firing patterns. These bifurcations were found to be similar to those simulated using the Hindmarsh-Rose model across two separated chaotic regions. One chaotic region lay between period-1 and period-2 burstings. This region has not attracted much attention. The other region is a well-known comb-shaped chaotic region, and it appears after period-2 bursting. After period-2 bursting, the chaotic firings lay in a period-adding bifurcation scenario or in a period-doubling bifurcation cascade. The deterministic dynamics of the chaotic firing patterns were identified using a nonlinear prediction method. These results provided details regarding the processes and dynamics of bifurcation containing the chaotic bursting between period-1 and period-2 burstings and other chaotic firing patterns within the comb-shaped chaotic region. They also provided details regarding the relationships between different firing patterns in parameter space.

\section{Introduction}

Nonlinear dynamics have been instrumental in the improvement of human understanding of the dynamics of neural firing patterns, which have been shown to play important roles in information processing [1-3]. Chaotic neural firing patterns have been observed in various nervous systems, and the possible roles of chaotic firing patterns in nervous system have been discussed in many studies [4-9]. Bifurcation scenarios including chaotic and periodic firing patterns have been simulated in many theoretical neuronal models, which have provided details regarding the relationships between different firing patterns in the parameter spaces [10-17]. The dynamics of physiological and pathological neural firing patterns and the possible roles have been studied using this knowledge of bifurcation structure of neural firing patterns [2].

Three-dimensional differential equations containing fast and slow variables have been used in investigations of bifurcations and chaos of neural firing patterns [10-15]. The
HR model has often been used to assess the chaos and bifurcations of both single neurons [11-13] and neuronal networks $[18,19]$. The HR model is composed of three nonlinear ordinary differential equations $[11-13,20]$, described as follows:

$$
\begin{gathered}
\frac{d x}{d t}=y-a x^{3}+b x^{2}-z+I, \\
\frac{d y}{d t}=c-d x^{2}-y, \\
\frac{d z}{d t}=r\left[s\left(x-x_{0}\right)-z\right] .
\end{gathered}
$$

Here, the variable $x$ represents the membrane potential, and $y$ and $z$ are the recovery and the slow adaption current, respectively. The model has eight parameters: $a, b, c, d, I, r$, $s$, and $x_{0}$. More detailed descriptions of the HR model can be found in previous studies $[11-13,20]$. In the present study, $a=1, b=3, c=1, d=5, s=4$, and $x_{0}=-1.6$. 


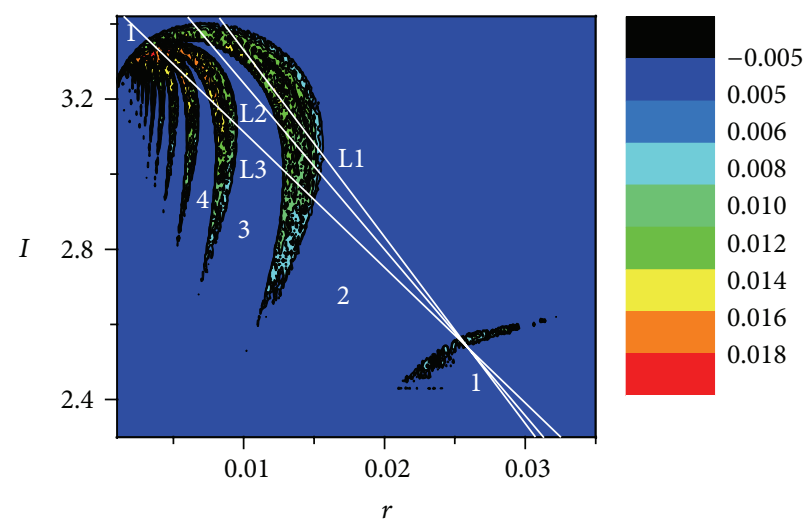

FIgURE 1: (Color online) the largest Lyapunov exponent in the $r \times I$ parameter space of the HR model $(0.001<r<0.035,2.3<I<3.42)$.

The largest Lyapunov exponent of firing patterns of the HR model in the $r \times I$ parameter space is shown in Figure 1 . There exist two separate chaotic regions. The first is the wellknown comb-shaped chaotic region [16, 17, 21-24], shown in the upper-left corner $(r<0.016$ and $I>2.6)$. The other is shown in the lower-right corner $(r>0.016$ and $I<2.6)$ between the period-1 and period-2 burstings [11]. Periodic firing patterns appear around the two chaotic regions. Almost all chaos simulated in the $\mathrm{HR}$ model in the previous investigations, like that induced by perioddoubling bifurcations and by intermittency, is located within the comb-shaped region. The complex bifurcation scenario from period-1 bursting to period-1 spiking was simulated as parameter $r$ decreased or $I$ increased across the comb-shaped chaotic region $[11-13,22]$. The complex processes show three components. First, a bifurcation of bursting patterns took place. Next, a transition from chaotic bursting to chaotic spiking was observed. Finally, an inverse period-doubling bifurcation from period-2 spiking to period-1 spiking was observed.

Theoretically, bursting and spiking patterns in the HR model can be distinguished using dissection of the fast and slow subsystems [25-29]. The first two equations, which had fast time scales, served as the fast subsystem. The third equation, which had a slow time scale, served as the slow subsystem. A classification scheme suitable for defining many kinds of bursting patterns and distinguishing bursting patterns from spiking patterns was built based on the combinations of bifurcations in the fast and slow subsystems [2529]. In appearance, bursting patterns are often characterized by alternations between bursts of continuous spikes and subsequent periods of quiescence, but spiking patterns have fast spikes without a period of quiescence. For example, the most obvious characteristic of the transition from the chaotic bursting to chaotic spiking is a sharp decrease in the interspike interval (ISI), which corresponds to the period of quiescence [30-32]. Spiking patterns usually appear in the region with small $r$ value or large $I$ value, and bursting patterns appear at other regions in the $r \times I$ parameter space. The number 1 in the upper-left corner of Figure 1 represents period-1 spiking and the other numbers, $1,2,3$, and 4 in the lower-left corner, represent period-1, $-2,-3$, and -4 burstings.

As shown in Figure 1, bifurcation scenarios from period-1 bursting to period-1 spiking across the two separated chaotic regions, like the behaviors along lines L1, L2, and L3, are not simulated in the HR model or observed in biological experiments. In previous biological experiments, which were performed on neural pacemakers, the bifurcation scenarios from period-1 bursting to period-1 spiking with chaotic firings, which locate within the comb-shaped chaotic region, were observed $[32,33]$. These bifurcations did not contain chaotic bursting between period-1 and period-2 burstings. Two examples of the chaotic firing between period-1 and period-2 firings were observed in previous experimental investigations $[34,35]$. One bifurcation scenario terminated at the period-2 bursting [34]. The other scenario lasted from period-1 bursting to chaotic bursting to period-2 firing to period-1 spiking [35]. These two experimental examples did not contain chaotic firing within the comb-shaped chaotic region.

In the present study, the experimental neural pacemaker employed in previous studies served as the experimental model [32-41]. Biological experimental observations of bifurcation scenarios are provided across two separate chaotic regions, which were similar to those reproduced using the HR model along lines L1, L2, and L3. The deterministic dynamics of the chaotic firing patterns were identified using the nonlinear time series analysis method. The experimental results demonstrated the existence of bifurcation scenarios containing two chaotic regions and chaotic burstings between period-1 and period-2 burstings in the real nervous system, which established the relationship between the two chaotic regions and between chaotic and periodic firing patterns in parameter space. The possible physiological and pathological roles of the bifurcations and chaos of the neural firing patterns in the neural pacemaker are discussed.

The rest of this paper is organized as follows. Simulation results of the HR model are reproduced in Section 2. Section 3 presents the experimental model. The experimental results are provided in Section 4 . Section 5 presents discussion and conclusion. 


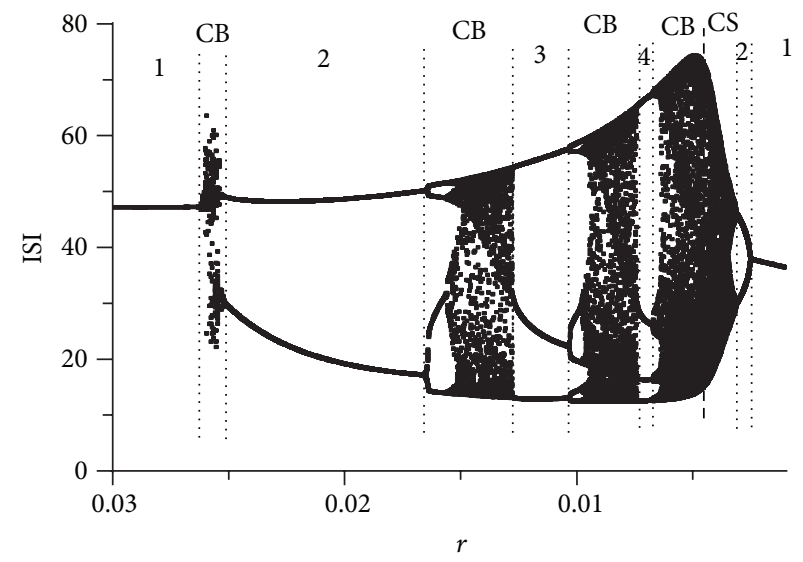

(a)

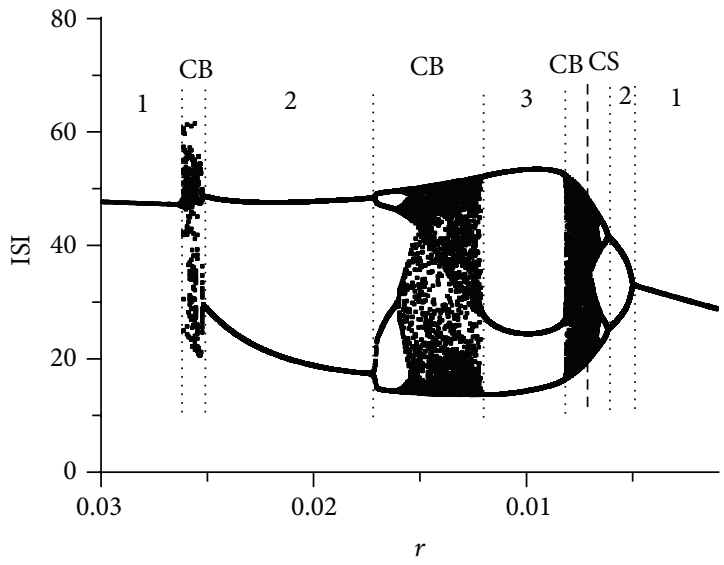

(b)

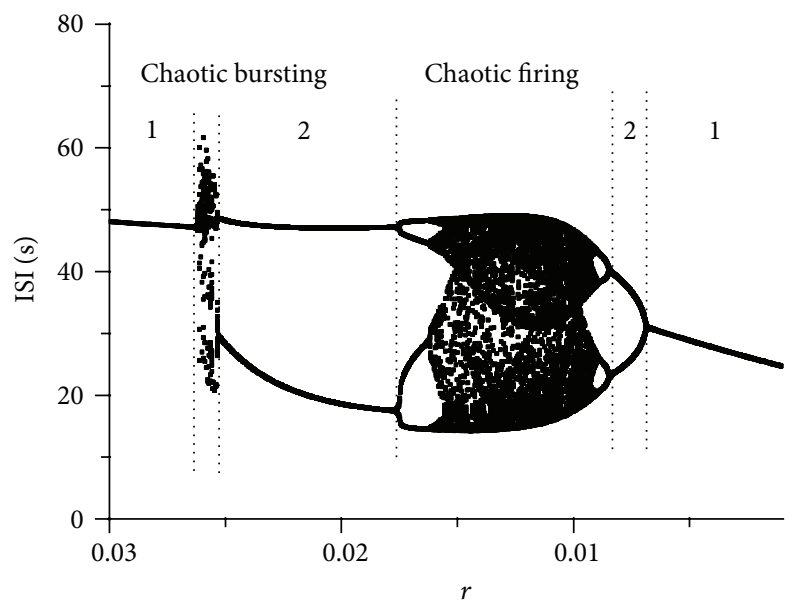

(c)

FIgURE 2: Bifurcation processes of the ISI series of firing patterns simulated using the HR model along lines shown in Figure $1(0.001<r<$ 0.03). (a) Line L3: $I=-36 \times(r-0.0265)+2.53$. (b) Line L2: $I=-44 \times(r-0.0265)+2.53$. (c) Line L1: $I=-50 \times(r-0.0265)+2.53$.

\section{Simulation Results in the HR Model}

2.1. Bifurcation Processes from Period-1 Bursting to Period1 Spiking. As shown in Figure 1, to explore a bifurcation scenario from period-1 bursting to period-1 spiking across two chaotic regions, the parameter $r$ must be decreased from a large value to a small one, and $I$ must be increased from a small value to a large one. Three examples of bifurcation scenarios resembling those along lines L1, L2, and L3 are provided as representatives to simulate the experimental results of Section 4. If $r$ and $I$ are chosen as different values, bifurcation scenarios similar to or different from the three examples can be simulated. We do not address other bifurcation processes.

The bifurcation scenario of example 1 lasts from period-1 bursting to chaotic bursting to period-2 bursting to chaotic bursting to period-3 bursting to chaotic bursting to period4 bursting to chaotic bursting to chaotic spiking to period-2 spiking to period-1 spiking as $r$ and $I$ change from the lowerright to the upper-left of the figure along line L3 with the following equation: $I=-36 \times(r-0.0265)+2.53(0.001<$ $r<0.03)$. The bifurcations of interspike intervals (ISIs) are shown in Figure 2(a). The thin dashed vertical lines separate the different chaotic and periodic patterns. The numbers represent the periods of the periodic firing patterns. The abbreviation $\mathrm{CB}$ represents chaotic bursting and CS represents chaotic spiking. The bold dashed vertical line approximately separates the chaotic bursting and chaotic spiking. The longest ISIs manifest a drastic decrease when chaotic bursting changed to chaotic spiking.

The bifurcation scenario shown in example 2 resembles the behavior along line L2 with the following equation: $I=$ $-44 \times(r-0.0265)+2.53(0.001<r<0.03)$. As $r$ and $I$ change from the lower-right to upper-left regions, the detailed processes are from period-1 bursting to chaotic bursting to period-2 bursting to chaotic bursting to period3 bursting to chaotic bursting to chaotic spiking to period-2 spiking to period-1 spiking, as shown in Figure 2(b).

The last example of bifurcation is similar to the behavior observed along line L1 with the following equation: $I=$ $-50 \times(r-0.0265)+2.53(0.001<r<0.03)$. As $r$ decreases, the processes are from period-1 bursting to chaotic bursting to period-2 bursting to chaotic bursting to chaotic spiking to period-2 spiking to period-1 spiking, as shown in 
Figure 2(c). The transition from chaotic bursting to chaotic spiking manifested a less drastic decrease than L3 and L2. No distinction was drawn between bursting and spiking patterns only according to the ISI values, both of which were here labeled as chaotic firing because the differences between bursting and spiking pattern are not the focus of the present study.

\subsection{Chaotic Firing Lying between Period-1 Bursting and} Period-2 Bursting. The spike trains of the chaotic bursting ( $I=2.53$ and $r=0.0245$ ) between period-1 bursting and period-2 bursting manifested irregular characteristics, as shown in Figure 3(a). The first return map of the ISI series exhibited a deterministic structure, as depicted in Figure 3(b). The deterministic chaotic firings within the comb-shaped chaotic region were examined in previous studies [11-13]. In the present study, no other chaotic firings were simulated in the HR model.

\section{Experimental Model}

3.1. Experimental Model. Bennet and Xie developed an animal model of chronic constriction injury (CCI) of the rat sciatic nerve [42]. The CCI model appears to reproduce many features of neuropathic pain disorders and can generate spontaneous neural firing [43]. This model has been adopted as a pacemaker to investigate the bifurcation and chaos of firing patterns [32-41].

An experimental neural pacemaker was formed at the site of injury of a rat sciatic nerve [42]. Surgery was performed to produce a pacemaker based on an injury induced by chronic ligature in adult male Sprague-Dawley rats (150-300 g). After a survival time of 6-14 days, the site of injury was exposed and perfused continuously with $34^{\circ} \mathrm{C}$ Krebs solution, in which a controlling extra-cellular calcium concentration $\left(\left[\mathrm{Ca}^{2+}\right]_{0}\right)$ is $1.2 \mathrm{mmol} / \mathrm{L}(\mathrm{mM})$. The spike trains of the spontaneous firing generated in the membrane at the site of injury were recorded from the individual fibers ending at the site of injury using a PowerLab system (ADInstruments, Australia) at a sampling frequency of $10.0 \mathrm{kHz}$. The neural pacemaker was used to investigate the bifurcations of firing patterns in a number of studies by adjusting the solution with different extra-cellular ionic concentration. A neural pacemaker often generates period-1 bursting patterns under controlled conditions, and previous studies have shown several bifurcations beginning from period-1 bursting with decreasing $\left[\mathrm{Ca}^{2+}\right]_{\mathrm{o}}[32-41]$.

In the present study, a neural pacemaker capable of generating period-1 bursting under controlled conditions was selected. The solution was replaced with $0 \mathrm{mM}\left[\mathrm{Ca}^{2+}\right]_{0}$. Although the replacement is sudden, the changes in the ion concentrations on the membrane of the neural pacemaker are gradual and continuous. The membrane dynamics can be changed slowly enough to facilitate evaluation of the transition and fast enough to produce a firing pattern different from the initial pattern within a finite time span. The protocol to adjust the bifurcation parameter is a practical and feasible method that has already seen considerable use in biological experimentation involving neural pacemakers and in investigations of other nervous systems [44-46].

3.2. Parameter Correspondence between the HR and Experimental Models. From a physiological perspective, multiple ionic currents including sodium, potassium, and calcium currents participate in the electrophysiology of nervous system. Potassium current that can induce the decrease or repolarization of the membrane potential participates in the generation of action potential through cooperation and competition with sodium current, which can induce the increase or depolarization of the membrane potential. Calcium current is a slow factor and can adjust the interval of continuous action potentials, that is, the ISIs, through the calcium-dependent potassium current which is related to the calcium concentration and/or the conductance of calciumdependent potassium channel. The modulation in $\left[\mathrm{Ca}^{2+}\right]_{\mathrm{o}}$ is a common method used to adjust neural firing patterns.

To a certain extent, $\left[\mathrm{Ca}^{2+}\right]_{\mathrm{o}}$ can adjust the ISIs of neural pacemakers, corresponding to the parameter $r$ related to the slow component of the HR model [47, 48]. Decreasing $\left[\mathrm{Ca}^{2+}\right]_{0}$ induced decreases in calcium-dependent potassium current, which increased the membrane potential $[47,48]$. These effects were very similar to those of increasing $I$ in the HR model. In this way, decreasing $\left[\mathrm{Ca}^{2+}\right]_{0}$ induced decreases in $r$ and increases in $I$ in the HR model. Therefore, $r$ and $I$ are changed dependently in Section 2 to simulate the decrease of $\left[\mathrm{Ca}^{2+}\right]_{\mathrm{o}}$ in the experiment.

\section{Experimental Results}

4.1. Overview of Experimental Bifurcations. In previous studies, most of experimental neural pacemakers exhibited bifurcation processes not invovling from period-1 bursting to period-1 spiking and only some pacemakers generated bifurcations from period-1 bursting to period-1 spiking [3241]. In the present study, forty-nine neural pacemakers generated bifurcations from period-1 bursting to period-1 spiking and 152 pacemakers manifested bifurcation processes not involving spiking patterns but including period-doubling to chaotic bursting, period-adding bifurcations with chaotic bursting, and period-adding sequences with stochastic bursting patterns. Some 23 out of 49 pacemakers manifested complex bifurcations from period-1 bursting to period-1 spiking with chaotic bursting. Bifurcation processes from period-1 bursting to period-1 spiking with chaotic bursting between period-1 bursting and period-1 spiking and chaotic firing patterns within the comb-shaped chaotic region were observed in 5 out of these 23 pacemakers. Two, two, and one out five neural pacemakers generated bifurcation processes similar to those simulated in the HR model along lines L3, L2, and L1, respectively. Another 14 out 152 pacemakers generated the bifurcation processes involving chaotic bursting between period-1 and period-2 burstings. The present study focused on the 5 bifurcation processes from period-1 bursting to period-1 spiking with chaotic bursting between period-1 bursting and period-1 spiking and chaotic firing patterns 


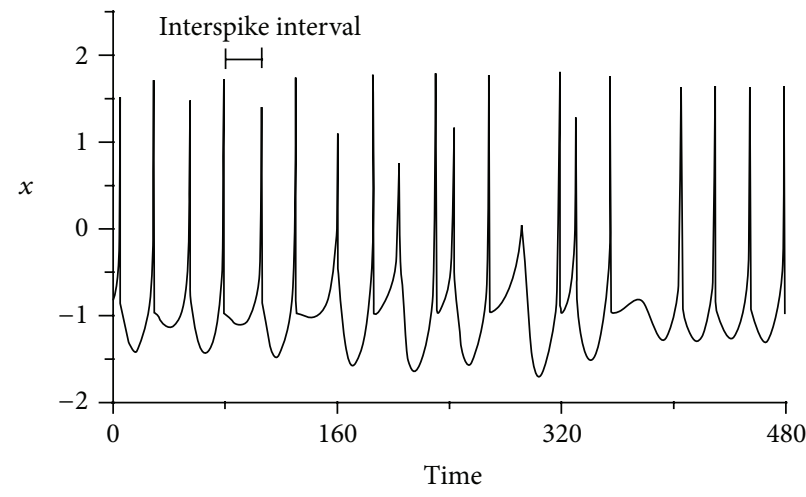

(a)

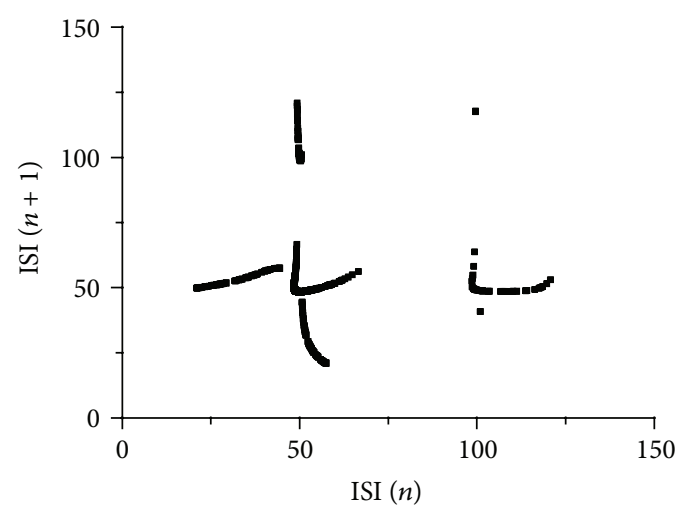

(b)

FIgURE 3: Chaotic firing pattern between period-1 bursting and period-2 bursting simulated in the HR model with $r=0.0245$ and $I=2.53$. (a) Spike trains. (b) The first return map of the ISI series.

within the comb-shaped chaotic region, and 3 examples are provided.

4.2. Three Examples of Bifurcation Scenarios. The bifurcation process of example 1 was similar to that simulated in the HR model along L3. The details involve period-1 bursting to chaotic bursting to period-2 bursting to chaotic bursting to period-3 bursting to chaotic bursting to period- 4 bursting to chaotic bursting to chaotic spiking to period2 spiking to period-1 spiking, as shown in Figure 4(a). The thin dashed vertical lines separate the different chaotic and periodic patterns. The numbers represent the periods of the periodic firing patterns. The abbreviation CB represents chaotic bursting and CS represents chaotic spiking. The process from period-2 bursting to chaotic bursting to period3 bursting to chaotic bursting to chaotic bursting is illustrated in Figure 4(b), manifesting a period-adding sequence with chaos. The transition from chaotic bursting to chaotic spiking exhibited a drastic decrease in the longest ISIs, as shown by the arrow and bold line in Figure 4(c). The first return map of ISI series of chaotic bursting between period-1 and period-2 burstings was similar to that of the HR model shown in Figure 3(b), as illustrated in Figure 5(a). The first return maps of ISI series of chaotic bursting between period-2 and period-3 burstings, the chaotic bursting before the drastic transition, and the chaotic spiking after the drastic transition are given in Figures 5(b), 5(c), and 5(d), respectively. All of the return maps manifested deterministic structures. The size of the chaotic spiking is much smaller than that of the chaotic bursting because of the drastic decrease in ISI values when chaotic bursting changed to chaotic spiking.

Example 2 of bifurcation scenario from period- 1 bursting to period-1 spiking observed from a pacemaker with decreasing $\left[\mathrm{Ca}^{2+}\right]_{0}$ was shown in Figure 6(a). In detail, the process lasted from period-1 bursting to chaotic bursting to period-2 bursting to chaotic bursting to period-3 bursting to chaotic bursting to chaotic spiking to period-2 spiking to period-1 spiking. The thin dashed vertical lines separate the different chaotic and periodic patterns. The numbers represent the periods of the periodic firing patterns. The abbreviation $\mathrm{CB}$ represents chaotic bursting and CS represents chaotic spiking. The bold dashed line distinguishes the chaotic bursting and spiking patterns. The process from chaotic bursting to chaotic spiking is shown in Figure 6(b). The bifurcation scenario manifested process similar to those simulated in the HR model as $r$ decreased along line L2, as shown in Figure 2(b). The first return map of ISI series of chaotic bursting between period- 1 bursting and period- 2 bursting is shown in Figure 7(a), similar to that simulated using the HR model. The first return map of ISI series of chaotic bursting after period-2 bursting manifested a deterministic structure, as shown in Figure 7(b). The first return map of ISI series of chaotic spiking also exhibited a deterministic structure, as depicted in Figure 7(c).

The bifurcation process shown in example 3 lasts from period-1 bursting to chaotic-bursting to chaotic spiking to period-2 spiking to period-1 spiking, as shown in Figure 8(a). The dashed vertical lines separate the different chaotic and periodic patterns. The numbers represent the periods of the periodic firing patterns. The chaotic firing pattern included the chaotic bursting and spiking, similar to that shown in Figure 2(c). The result shows that the period-adding sequences resemble those simulated with the HR model with decreasing $r$ along line L1. The first return map of ISI series of the chaotic bursting between period-1 and period2 burstings manifested only a part of that of HR model shown in Figure 3(b), which was similar to that observed in a previous study [34], as illustrated in Figure 8(b). The first return map of the chaotic firing lying between period2 bursting and period-2 spiking exhibited a deterministic structure, as shown in Figure 8(c).

4.3. Deterministic Characteristics of the Chaotic Firing Patterns. The deterministic property of chaotic firing patterns can be estimated by nonlinear time series analysis method $[34,37,49,50]$. In this study, the normalized prediction error (NPE) was computed using the simple nearest-neighbor method $[49,50]$ with the following algorithm. 


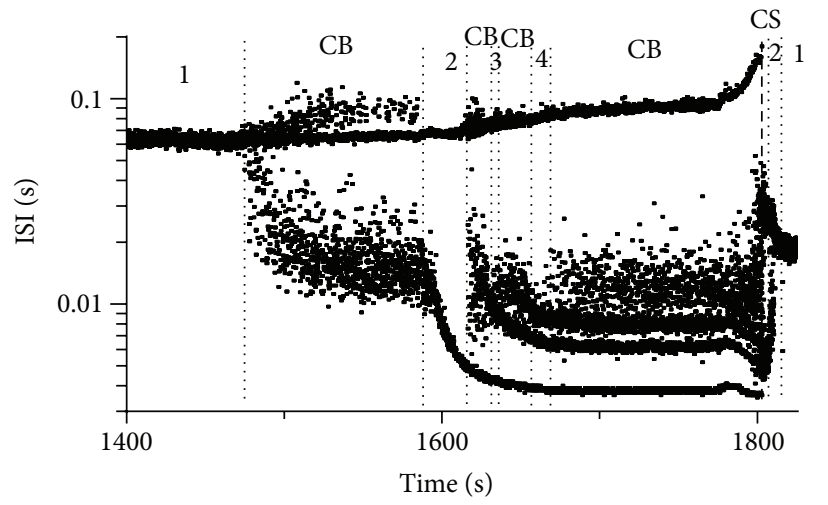

(a)

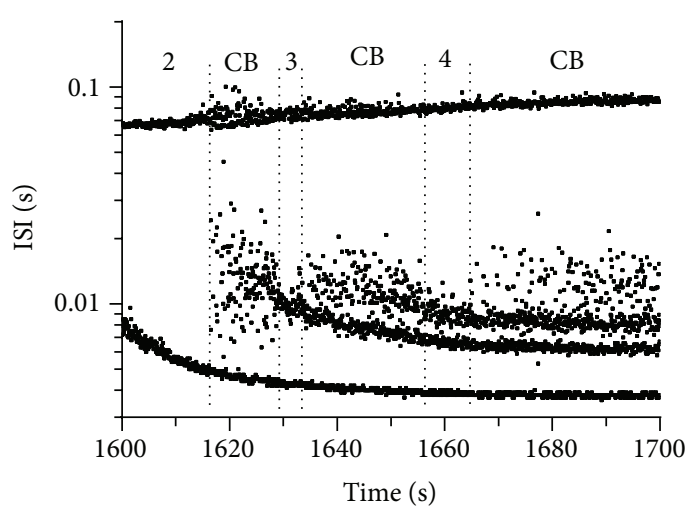

(b)

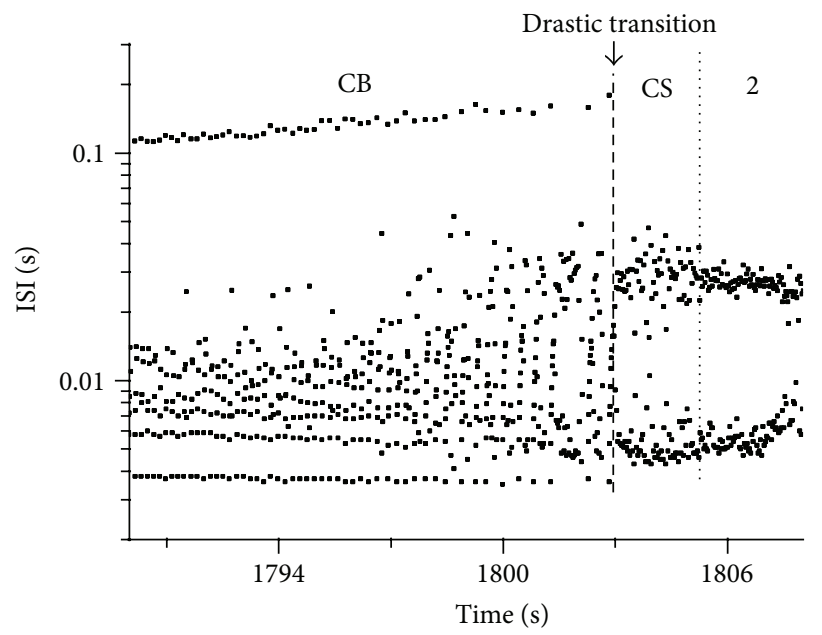

(c)

FIGURE 4: Example 1 of bifurcation process from period-1 bursting to period-1 spiking. (a) In detail, the process lasts from period-1 bursting to chaotic bursting to period-2 bursting to chaotic bursting to period-3 bursting to chaotic bursting to period- 4 bursting to chaotic bursting to chaotic spiking to period-2 spiking to period-1 spiking. (b) Detail of Figure 4(a), which involves period-adding bifurcation with chaos from period-2 bursting to chaotic bursting after period-4 bursting. (c) Detail of Figure 4(a), which involves chaotic bursting to chaotic spiking to period-2 spiking.

With a reconstruction dimension $m$, a time series $t_{i}(i=$ $1,2, \ldots, L)$ is transformed to $L-m+1$ state points in space $R^{m}$. For a point $V_{n}=\left(t_{n}, t_{n+1}, \ldots, t_{n+m-1}\right) \quad(n=1,2, \ldots, L-m+1)$ in space $R^{m}, M=\beta(L-m+1)$ points nearest to $V_{n}$ are chosen and written as $U_{k}=\left(t_{j_{k}}, \ldots, t_{j_{k}+m-1}\right)(1 \leq k \leq$ $M)$. The average $p(n, h)=(1 / M) \sum_{k=1}^{M} t_{j_{k}+h}$ is then used to approximate the future value $t_{n+h}$. The difference $p(n, h)-t_{n+h}$ is the $h$ th step prediction error for point $V_{n}$. For all state points, the normalized prediction error (NPE) is defined as follows:

$$
\operatorname{NPE}(h)=\frac{\left((1 /(L-m+1)) \sum_{n=1}^{L-m+1}\left(p(n, h)-t_{n+h}\right)^{2}\right)^{1 / 2}}{\left((1 /(L-m+1)) \sum_{n=1}^{L-m+1}\left(\bar{t}-t_{n+h}\right)^{2}\right)^{1 / 2}},
$$

where $\bar{t}$ is the average of time series $t_{i}$. From the definition, a value of NPE much less than 1.0 means that there is linear or nonlinear predictability in the time series beyond the baseline prediction of the series mean. In general, the NPE of raw data and surrogate data are compared. The surrogate data generated by random shuffle method, shuffling the original sequence randomly, have the same probability distribution as the original data, but the deterministic temporal structures within ISI series are broken [51]. The NPE value of the original chaotic ISI series is much less than 1 in any short-term prediction and approaches 1 in the long term. However, the surrogate data is always approximately equal to 1 , showing the deterministic property of the original series.

All experimental chaotic firings exhibited a short-term prediction when $m=3,4$, or 5 and $\beta=0.5 \%, 1 \%$, or $2 \%$. Four examples are provided as representative. The NPE ( $m=4$ and $\beta=1 \%$ ) of the chaotic bursting between period-1 and period- 2 burstings of example 1 (Figure 5(a)), the chaotic bursting between period- 2 and period- 3 burstings of example 2 (Figure $7(\mathrm{~b})$ ), the chaotic spiking of example 2 (Figure $7(\mathrm{c})$ ), the chaotic firing pattern between period-1 and period-2 burstings of example 3 (Figure 8(b)), and the corresponding 


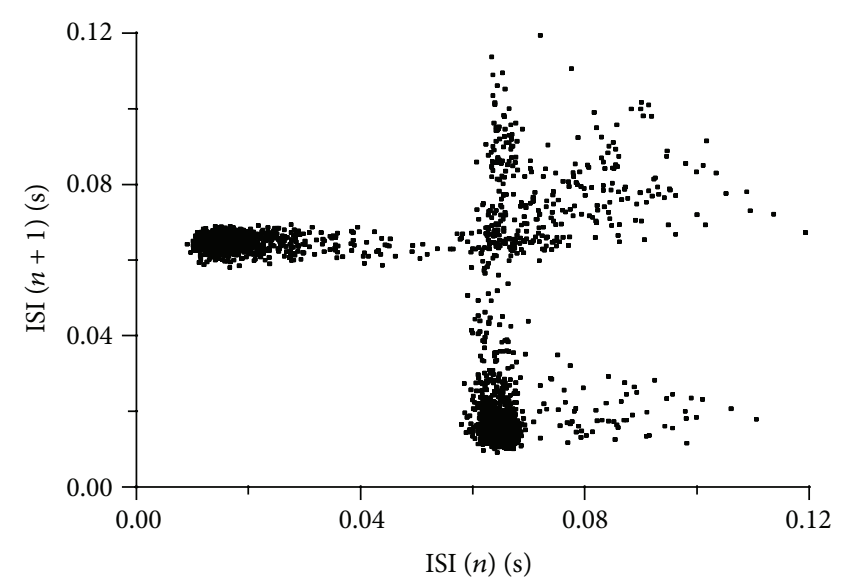

(a)

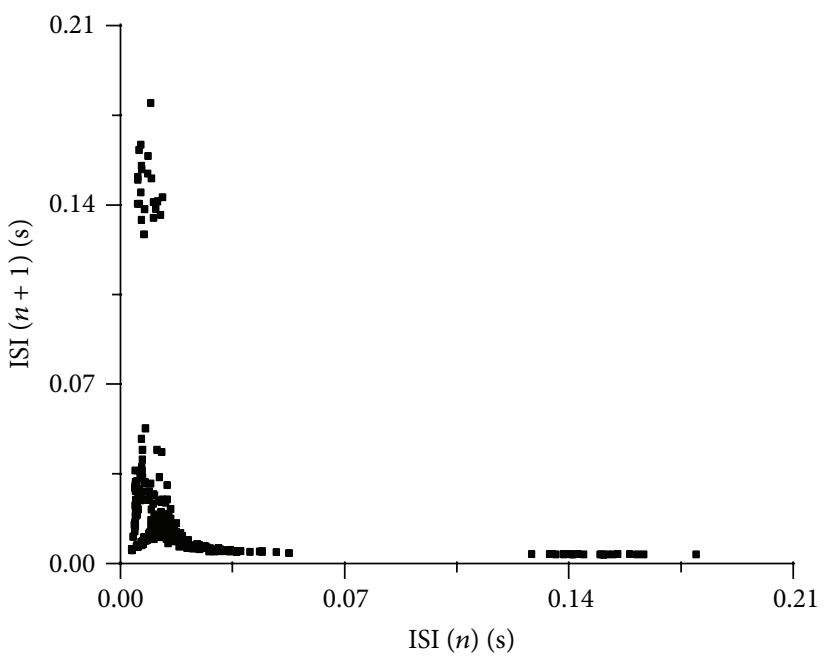

(c)

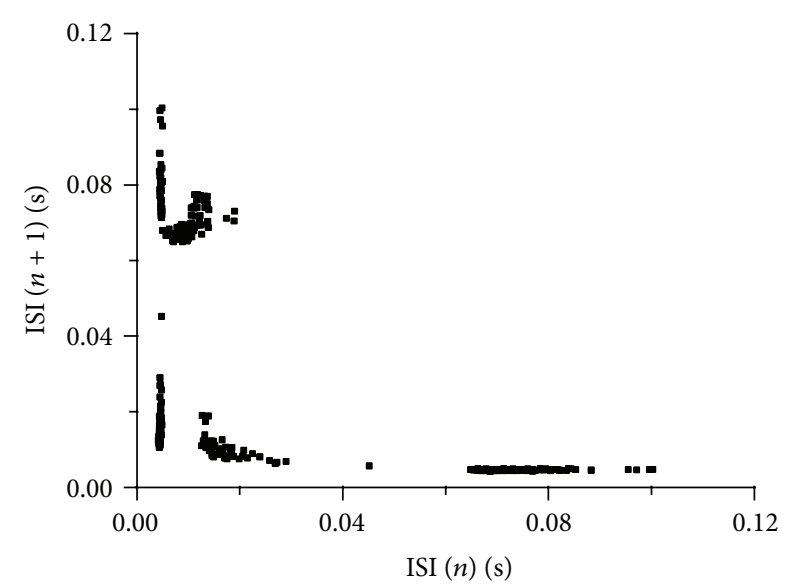

(b)

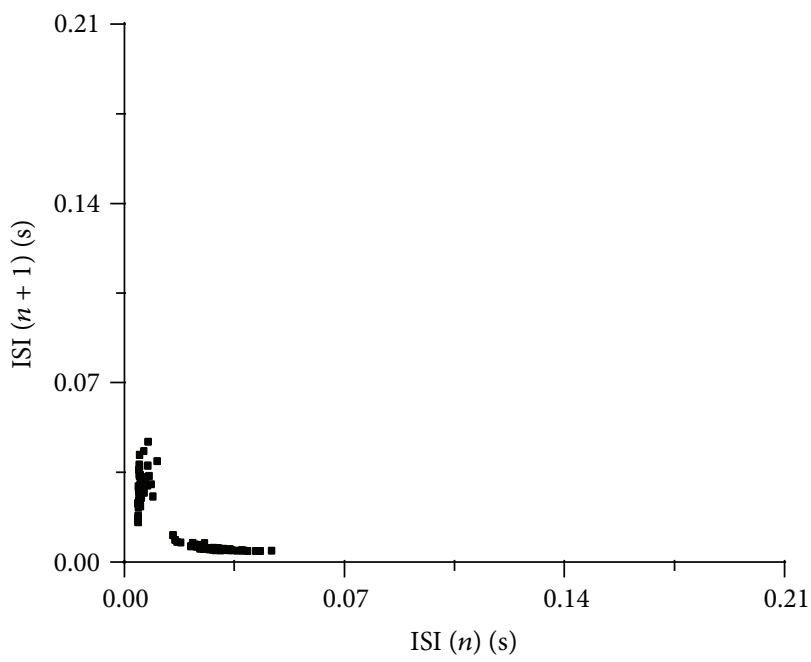

(d)

FIGURE 5: The first return map of ISI series of chaotic firing within the bifurcation scenario shown in Figure 4. (a) Chaotic bursting lying between period-1 and period- 2 bursting patterns. (b) Chaotic bursting between period- 2 and period-3 bursting patterns. (c) Chaotic bursting after period-4 bursting. (d) Chaotic spiking.

surrogate data (mean of 10 realizations) are shown in Figures 9(a)-9(d). The original chaotic firing exhibited a short-term prediction and the surrogate data manifested no predication. The results showed that the experimental chaotic firing patterns possessed deterministic dynamics.

\section{Conclusion and Discussion}

Bifurcation scenarios containing chaotic bursting between period-1 and period-2 burstings and chaotic firings appearing after period-2 bursting in period-adding sequences or period-doubling cascade were observed in biological experiments performed using different neural pacemakers. The deterministic dynamics within the chaotic bursting were identified. The experimental results manifested characteristics that were very similar to those of the HR model in parameter space. These characteristics included two chaotic regions; one is the well-known comb-shaped region appearing after period- 2 bursting and the other is the chaotic region between period- 1 and period- 2 burstings. The results showed the chaotic firing pattern between period-1 and period-2 bursting and bifurcation scenarios to contain two chaotic regions, such as those observed in real nervous systems. With exception of the two separated chaotic regions, the process that transitioned from period-1 bursting to period-1 spiking through complex processes should be emphasized. Most bifurcation processes observed from the neural pacemakers terminated at a certain firing pattern before period-1 spiking $[36,37,40,41]$. The complex bifurcation processes from period-1 bursting to period-1 spiking in the present paper contained the chaotic bursting lying between period-1 and period-2 burstings, different from those observed in our previous studies $[32,33]$, wherein no chaotic bursting lying between period- 1 and period- 2 burstings was identified. The experimental result provides novel examples of complex bifurcation process from period-1 bursting to period-1 spiking. The experimental observations showed the relationships between chaotic burstings lying within two chaotic regions and between bursting and spiking patterns, which is important for the identification of different chaotic firing patterns 


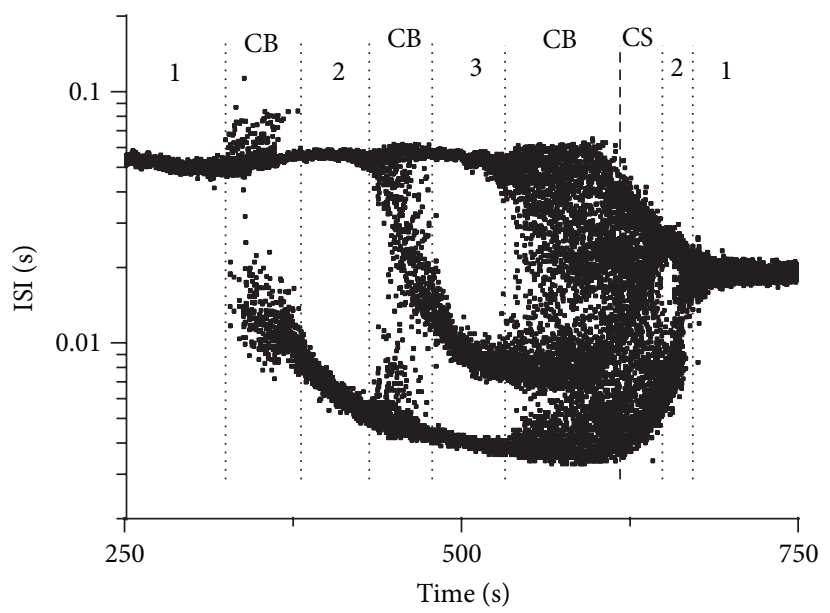

(a)

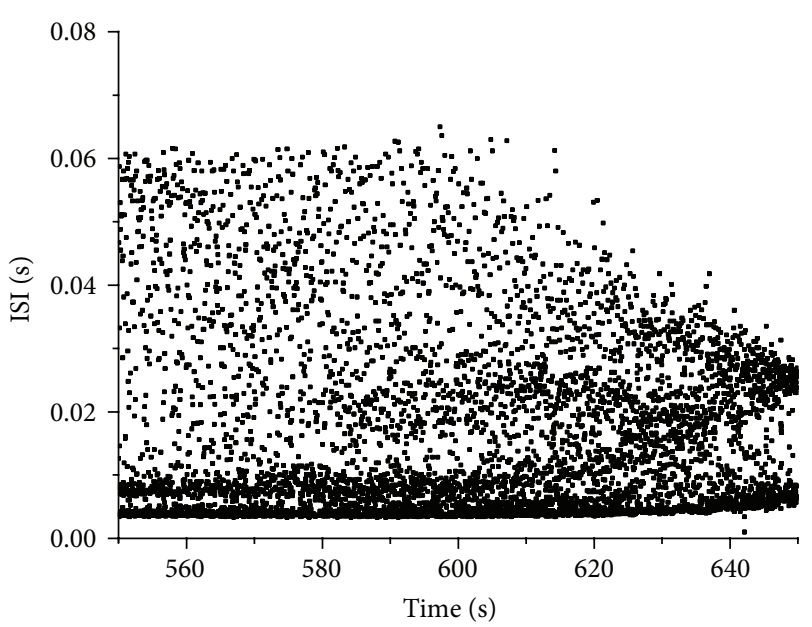

(b)

FIGURE 6: Example 2 of bifurcation scenario as observed from a pacemaker with decreasing $\left[\mathrm{Ca}^{2+}\right]_{0}$. (a) Bifurcation scenario from period-1 bursting to period-1 spiking. In detail, the process is from period-1 bursting to chaotic bursting to period-2 bursting to chaotic bursting to period-3 bursting to chaotic bursting to chaotic spiking to period-2 spiking to period-1 spiking. (b) Part of Figure 6(a); the process is from chaotic bursting to chaotic spiking.

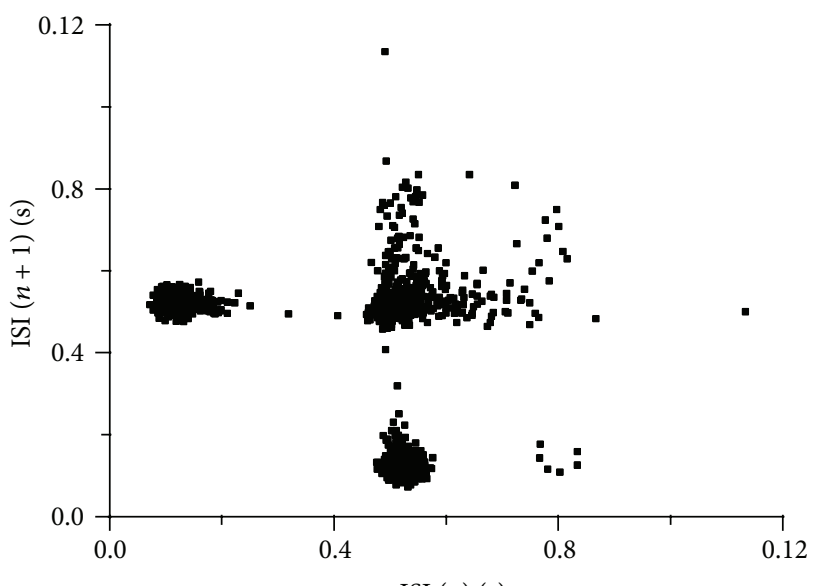

ISI $(n)(s)$

(a)

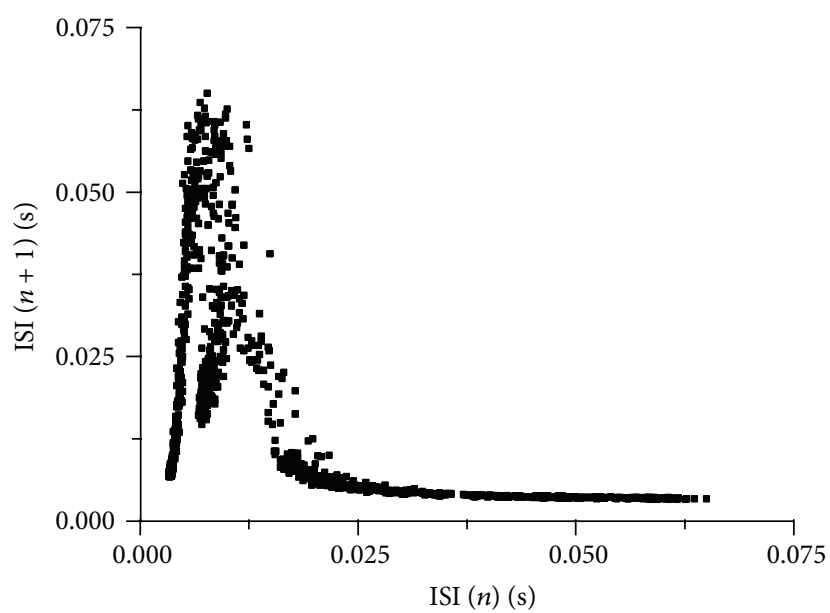

(b)

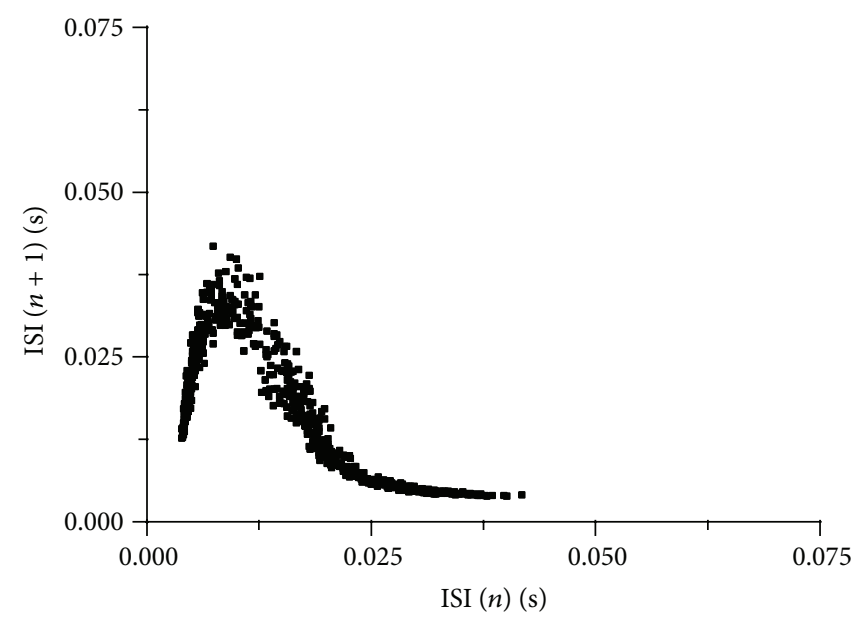

(c)

FIgURE 7: The first return maps of ISI series of chaotic firings within the bifurcation scenario as shown in Figure 6(a). (a) The chaotic bursting between period-1 and period-2 bursting patterns. (b) The chaotic bursting appearing after period-3 bursting patterns. (c) Chaotic spiking. 


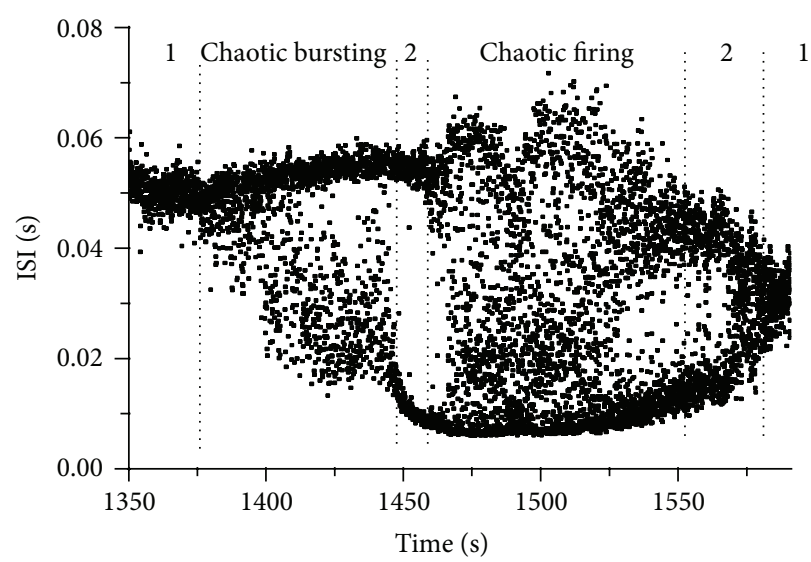

(a)

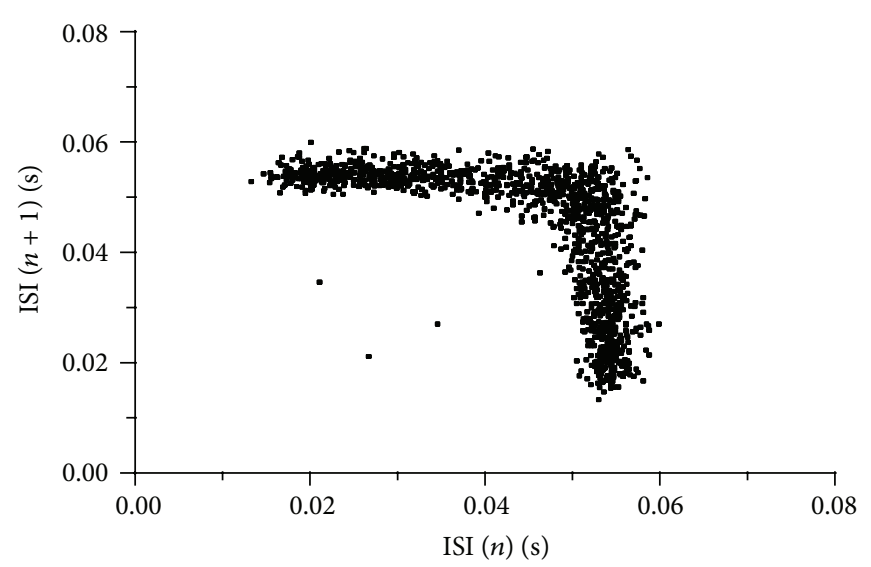

(b)

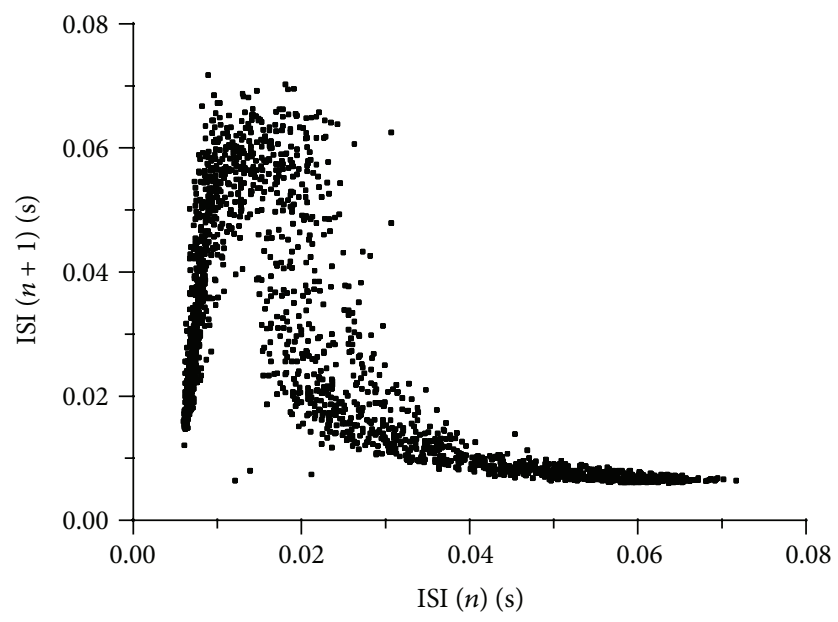

(c)

FIGURE 8: Example 3 of bifurcation scenario and firing patterns observed from a pacemaker with decreasing $\left[\mathrm{Ca}^{2+}\right]_{0}$. (a) Bifurcation scenario from period-1 bursting to chaotic bursting to period-2 bursting to chaotic firing to period-2 spiking to period-1 spiking. (b) The first return map of ISI series of the chaotic firing between period-1 and period-2 burstings. (c) The first return map of ISI series of the chaotic firing.

and the relationships between periodic and chaotic firing patterns in parameter space.

As studied in a previous study [52], neural firing patterns existing within a short parameter range have become blurred and disappeared in the bifurcations, influenced by noise of a suitable intensity. Considering that noise is inevitable in the real nervous system, the firing patterns existing in a narrow parameter region, such as the period- 4 bursting proceeding to period-2 bursting in Figure 2, disappeared in the experimental bifurcations, as shown in Figures 4(a), 6(a), and $8(a)$. Based on such a viewpoint, we can conclude that the experimental bifurcation closely matches those reproduced by the HR model. In addition, the chaotic bursting between period-1 bursting and period-2 bursting in example 3 was similar to the one observed in a previous study [34] but it was different from those in another previous study [35] and in examples 1 and 2. Experiments should be performed on more neural pacemakers to further identify the dynamics of chaos between period- 1 and period- 2 burstings.

Compared with many simulation results about the bifurcations and chaos of the HR model, there existed less theo- retical investigations of the HR model. The generation of the chaotic bursting and the transition from bursting to spiking in excitable membrane models were analyzed in a theoretical model resembling HR model by Terman [53, 54]. The different cases of period-adding bifurcations were distinguished and analyzed in both of the original HR model and a map reduced from the HR model [11-13]. The mechanism of the transition from the chaotic bursting to spiking was analyzed in several references $[21,30,31,54,55]$, suggested as a homoclinic reinjection to an unstable chaotic saddle, a continuous interior crisis or trajectory separatrix in the manifolds of the equilibrium point. The Floquet multipliers of periodic neural firing patterns were calculated numerically with a modified shooting method, and perioddoubling bifurcation point of bursting and spiking patterns and the tangent bifurcation point from period- 3 bursting to chaotic bursting were identified in our previous study [56]. Codimensional-2 bifurcation analysis was performed to investigate the dynamics of the period-adding bifurcations [57]. The generation of the chaotic bursting lying between period-1 and period- 2 burstings was suggested to be caused 


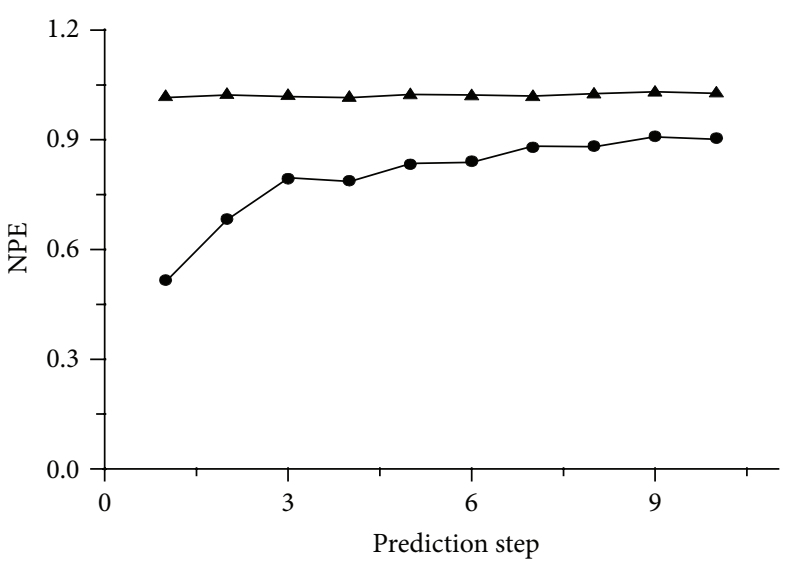

(a)

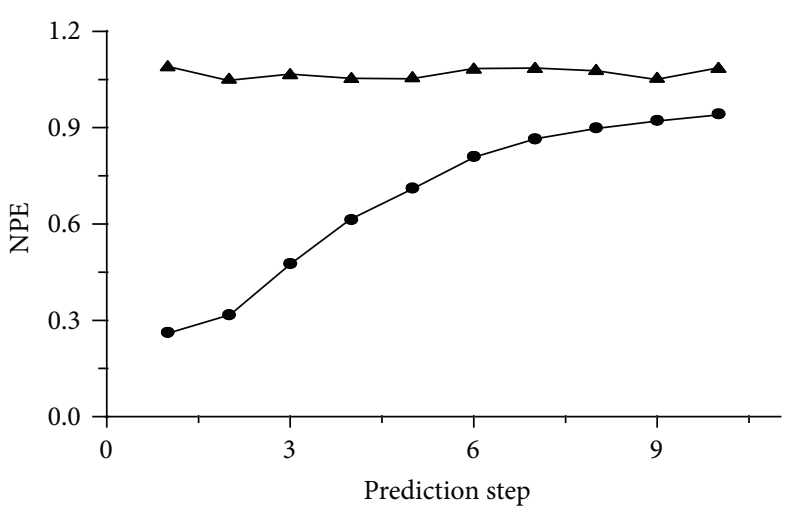

(c)

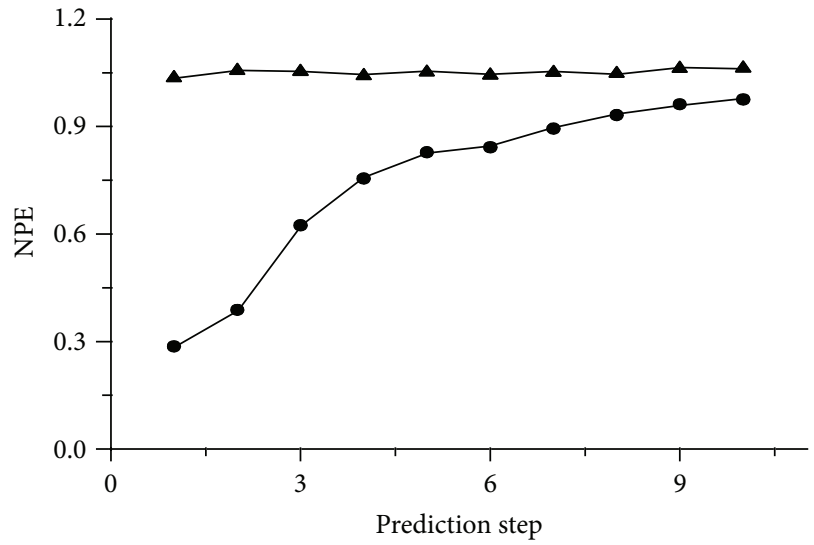

(b)

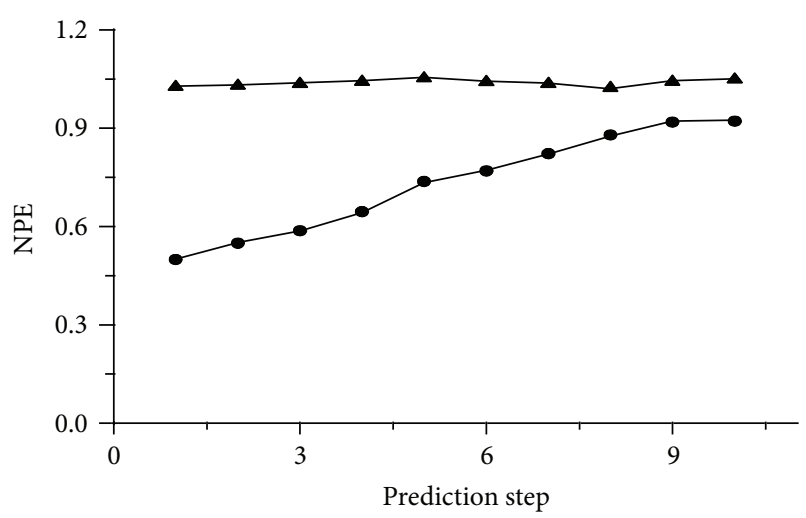

(d)

Figure 9: NPE of the experimental chaotic firings. (a) The chaotic bursting between period-1 and period-2 burstings of example 1 (Figure 5(a)). (b) The chaotic bursting lying between period-2 and period-3 burstings of example 2 (Figure 7(b)). (c) The chaotic spiking of example 2 (Figure $7(\mathrm{c})$ ). (d) The chaotic bursting lying between period-1 and period-2 burstings of example 3 (Figure 8 (b)). Lines with squares show the original chaotic firing pattern and lines with triangles show the surrogate data.

by a crisis by Holden and Fan [11], needed to be further investigated.

Neural firing patterns play important roles in neural information processing in different nervous systems [13]. Chaotic firing patterns have been observed in different nervous systems and the roles of chaotic behaviors have been discussed [32-41]. The CCI model has been widely used to investigate abnormal spontaneous pain [42, 43, 47, $48,52]$. Neural firing patterns were found to participate in the abnormal spontaneous pain. The responses of chaotic bursting to an external electronic stimulation have been reported as a critical sensitivity [9]. The details of the biological role of periodic and chaotic firing patterns of the CCI model may be investigated in the future using very difficult experimental designs through recording of animal behaviors, such as spontaneous foot lifting, at the same time as neural firing patterns [58].

\section{Conflict of Interests}

The authors declare that there is no conflict of interests regarding the publication of this paper.

\section{Acknowledgments}

This work was supported by the National Natural Science Foundation of China under Grant nos. 11372224 and 11072135 and the Fundamental Research Funds for Central Universities of Tongji University under Grant no. 1330219127.

\section{References}

[1] H. A. Braun, H. Wissing, K. Schäfer, and M. C. Hirsch, "Oscillation and noise determine signal transduction in shark multimodal sensory cells," Nature, vol. 367 , no. 6460 , pp. $270-$ 273, 1994.

[2] M. H. Yang, S. C. An, H. G. Gu, Z. Q. Liu, and W. Ren, "Understanding of physiological neural firing patterns through dynamical bifurcation machineries," NeuroReport, vol. 17, no. 10, pp. 995-999, 2006.

[3] J. M. González-Miranda, "Block structured dynamics and neuronal coding," Physical Review E, vol. 72, no. 5, Article ID 051922, 2005.

[4] H. Hayashi, S. Ishizuka, and K. Hirakawa, "Transition to chaos via intermittency in the onchidium pacemaker neuron," Physics Letters A, vol. 98, no. 8-9, pp. 474-476, 1983. 
[5] K. Aihara, G. Matsumoto, and Y. Ikegaya, "Periodic and nonperiodic responses of a periodically forced hodgkin-huxley oscillator," Journal of Theoretical Biology, vol. 109, no. 2, pp. 249269, 1984.

[6] T. Elbert, W. J. Ray, Z. J. Kowalik, J. E. Skinner, K. E. Graf, and N. Birbaumer, "Chaos and physiology: deterministic chaos in excitable cell assemblies," Physiological Reviews, vol. 74, no. 1, pp. 1-48, 1994.

[7] J. X. Xu, Y. F. Gong, W. Ren, S. J. Hu, and F. Z. Wang, "Propagation of periodic and chaotic action potential trains along nerve fibers," Physica D, vol. 100, no. 1-2, pp. 212-224, 1997.

[8] M. I. Rabinovich and H. D. I. Abarbanel, "The role of chaos in neural systems," Neuroscience, vol. 87, no. 1, pp. 5-14, 1998.

[9] J. Yang, Y. B. Duan, J. L. Xing, J. L. Zhu, J. H. Duan, and S. J. $\mathrm{Hu}$, "Responsiveness of a neural pacemaker near the bifurcation point," Neuroscience Letters, vol. 392, no. 1-2, pp. 105-109, 2006.

[10] T. R. Chay, "Chaos in a three-variable model of an excitable cell," Physica D, vol. 16, no. 2, pp. 233-242, 1985.

[11] A. V. Holden and Y. Fan, "From simple to complex oscillatory behaviour via intermittent chaos in the Rose-Hindmarsh model for neuronal activity," Chaos, Solitons and Fractals, vol. 2, no. 4, pp. 349-369, 1992.

[12] A. V. Holden and Y. Fan, "From simple to simple bursting oscillatory behaviour via chaos in the Rose-Hindmarsh model for neuronal activity," Chaos, Solitons and Fractals, vol. 2, no. 3, pp. 221-236, 1992.

[13] Y. S. Fan and A. V. Holden, "Bifurcations, burstings, chaos and crises in the Rose-Hindmarsh model for neuronal activity," Chaos, Solitons and Fractals, vol. 3, no. 4, pp. 439-449, 1993.

[14] Y. Fan and T. R. Chay, "Generation of periodic and chaotic bursting in an excitable cell model," Biological Cybernetics, vol. 71, no. 5, pp. 417-431, 1994.

[15] T. R. Chay, Y. S. Fan, and Y. S. Lee, "Bursting, spiking, chaos, fractals and universality in biological rhythms," International Journal of Bifurcation and Chaos in Applied Sciences and Engineering, vol. 5, no. 3, pp. 596-635, 1995.

[16] M. Storace, D. Linaro, and E. De Lange, "The HindmarshRose neuron model: bifurcation analysis and piecewise-linear approximations," Chaos, vol. 18, no. 3, Article ID 033128, 2008.

[17] R. Barrio and A. Shilnikov, "Parameter-sweeping techniques for temporal dynamics of neuronal systems: case study of Hindmarsh-Rose model," The Journal of Mathematical Neuroscience, vol. 1, no. 1, pp. 1-22, 2011.

[18] D. Hansel and H. Sompolinsky, "Synchronization and computation in a chaotic neural network," Physical Review Letters, vol. 68, no. 5, pp. 718-721, 1992.

[19] M. Dhamala, V. K. Jirsa, and M. Ding, "Transitions to synchrony in coupled bursting neurons," Physical Review Letters, vol. 92, no. 2, pp. 281011-281014, 2004.

[20] J. L. Hindmarsh and R. M. Rose, "A model of neuronal bursting using three coupled first order differential equations," Proceedings of the Royal Society of London B, vol. 221, no. 1222, pp. 87-102, 1984.

[21] G. Innocenti, A. Morelli, R. Genesio, and A. Torcini, "Dynamical phases of the Hindmarsh-Rose neuronal model: studies of the transition from bursting to spiking chaos," Chaos, vol. 17, no. 4, Article ID 043128, 2007.

[22] J. M. González-Miranda, "Complex bifurcation structures in the Hindmarsh-Rose neuron model," International Journal of bifurcation and Chaos in Applied Sciences and Engineering, vol. 17, no. 9, pp. 3071-3083, 2007.
[23] A. Shilnikov and M. Kolomiets, "Methods of the qualitative theory for the Hindmarsh-Rose model: a case study. A tutorial," International Journal of Bifurcation and Chaos in Applied Sciences and Engineering, vol. 18, no. 8, pp. 2141-2168, 2008.

[24] P. C. Rech, "Dynamics of a neuron model in different twodimensional parameter-spaces," Physics Letters A, vol. 375, no. 12, pp. 1461-1464, 2011.

[25] J. Rinzel, "A formal classification ofbursting mechanisms in excitable systems," in Mathematical Topics in Population Biology, Morphogenesis and Neurosciences, vol. 71 of Lecture Notes in Biomathematics, pp. 267-281, 1987.

[26] J. F. Nong, "Parameters selection and noise estimation of SVM regression," in Proceedings of the 5th International Joint Conference on Computational Sciences and Optimization, pp. 379-381, 2012.

[27] J. Rinzel and Y. S. Lee, "Dissection of a model for neuronal parabolic bursting," Journal of Mathematical Biology, vol. 25, no. 6, pp. 653-675, 1987.

[28] R. Bertram, M. J. Butte, T. Kiemel, and A. Sherman, "Topological and phenomenological classification of bursting oscillations," Bulletin of Mathematical Biology, vol. 57, no. 3, pp. 413439, 1995.

[29] E. M. Izhikevich, "Neural excitability, spiking and bursting," International Journal of Bifurcation and Chaos in Applied Sciences and Engineering, vol. 10, no. 6, pp. 1171-1266, 2000.

[30] J. M. González-Miranda, "Observation of a continuous interior crisis in the Hindmarsh-Rose neuron model," Chaos, vol. 13, no. 3, pp. 845-852, 2003.

[31] G. Innocenti and R. Genesio, "On the dynamics of chaotic spiking-bursting transition in the Hindmarsh-Rose neuron," Chaos, vol. 19, no. 2, Article ID 023124, 2009.

[32] H. G. Gu, "Biological experimental demonstration of transitions from the chaotic bursting to chaotic spiking," Chaos, vol. 23, no. 2, Article ID 023126, 2013.

[33] L. Li, H. G. Gu, M. H. Yang, Z. Q. Liu, and W. Ren, "A series of bifurcation scenarios in the firing pattern transitions in an experimental neural pacemaker," International Journal of Bifurcation and Chaos in Applied Sciences and Engineering, vol. 14, no. 5, pp. 1813-1817, 2004.

[34] Q. S. Lu, H. G. Gu, Z. Q. Yang, X. Shi, L. X. Duan, and Y. H. Zheng, "Dynamics of firing patterns, synchronization and resonances in neuronal electrical activities: experiments and analysis," Acta Mechanica Sinica, vol. 24, no. 6, pp. 593-628, 2008.

[35] X. B. Wu, J. Mo, M. H. Yang, Q. H. Zheng, H. G. Gu, and W. Ren, "Two different bifurcation scenarios in neural firing rhythms discovered in biological experiments by adjusting two parameters," Chinese Physics Letters, vol. 25, no. 8, pp. 2799$2802,2008$.

[36] H. G. Gu, M. H. Yang, L. Li, Z. Q. Liu, and W. Ren, "Dynamics of autonomous stochastic resonance in neural period adding bifurcation scenarios," Physics Letters A, vol. 319, no. 1-2, pp. 8996, 2003.

[37] M. H. Yang, Z. Q. Liu, L. Li et al., "Identifying distinct stochastic dynamics from chao: a Study on multimodal neural firing patterns," International Journal of Bifurcation and Chaos in Applied Sciences and Engineering, vol. 19, no. 2, pp. 453-485, 2009.

[38] H. G. Gu, M. H. Yang, L. Li, Z. Q. Liu, and W. Ren, "Experimental observation of the stochastic bursting caused by coherence 
resonance in a neural pacemaker," NeuroReport, vol. 13, no. 13, pp. 1657-1660, 2002.

[39] H. G. Gu, W. Ren, Q. S. Lu, S. G. Wu, M. H. Yang, and W. Chen, "Integer multiple spiking in neuronal pacemakers without external periodic stimulation," Physics Letters A, vol. 285, no. 1-2, pp. 63-68, 2001.

[40] H. G. Gu, B. Jia, and G. R. Chen, "Experimental evidence of a chaotic region in a neural pacemaker," Physics Letters A, vol. 377, no. 9, pp. 718-720, 2013.

[41] B. Jia, H. G. Gu, L. Li, and X. Y. Zhao, "Dynamics of perioddoubling bifurcation to chaos in the spontaneous neural firing patterns," Cognitive Neurodynamics, vol. 6, no. 1, pp. 89-106, 2011.

[42] G. J. Bennett and Y. K. Xie, "A peripheral mononeuropathy in rat that produces disorders of pain sensation like those seen in man," Pain, vol. 33, no. 1, pp. 87-107, 1988.

[43] M. Tal and E. Eliav, "Abnormal discharge originates at the site of nerve injury in experimental constriction neuropathy (CCI) in the rat," Pain, vol. 64, no. 3, pp. 511-518, 1996.

[44] H. A. Braun, J. Schwabedal, M. Dewald et al., "Noise-induced precursors of tonic-to-bursting transitions in hypothalamic neurons and in a conductance-based model," Chaos, vol. 21, no. 4, Article ID 047509, 2011.

[45] M. Y. Kim, M. Aguilar, A. Hodge, E. Vigmond, A. Shrier, and L. Glass, "Stochastic and spatial influences on drug-induced bifurcations in cardiac tissue culture," Physical Review Letters, vol. 103, no. 5, Article ID 058101, 2009.

[46] T. Quail, N. McVicar, M. Aguilar et al., "Chaotic dynamics in cardiac aggregates induced by potassium channel block," Chaos, vol. 22, no. 3, Article ID 033140, 2012.

[47] Y. K. Xie, "Mechanism for chronic pain generation," Chinese Science Bulletin, vol. 45, no. 9, pp. 775-783, 2000.

[48] L. C. Mongan, M. J. Hill, M. X. Chen et al., "The distribution of small and intermediate conductance calcium-activated potassium channels in the rat sensory nervous system," Neuroscience, vol. 131, no. 1, pp. 161-175, 2005.

[49] J. D. Farmer and J. J. Sidorowich, "Predicting chaotic time series," Physical Review Letters, vol. 59, no. 8, pp. 845-848, 1987.

[50] T. Sauer, "Reconstruction of dynamical systems from interspike intervals," Physical Review Letters, vol. 72, no. 24, pp. 3811-3814, 1994.

[51] J. Theiler, S. Eubank, A. Longtin, B. Galdrikian, and J. Doyne Farmer, "Testing for nonlinearity in time series: the method of surrogate data," Physica D, vol. 58, no. 1-4, pp. 77-94, 1992.

[52] U. Feudel, A. Neiman, X. Pei et al., "Homoclinic bifurcation in a Hodgkin-Huxley model of thermally sensitive neurons," Chaos, vol. 10, no. 1, pp. 231-239, 2000.

[53] D. Terman, "Chaotic spikes arising from a model of bursting in excitable membranes," SIAM Journal on Applied Mathematics, vol. 51, no. 5, pp. 1418-1450, 1991.

[54] D. Terman, "The transition from bursting to continuous spiking in excitable membrane models," Journal of Nonlinear Science, vol. 2, no. 2, pp. 135-182, 1992.

[55] X. J. Wang, "Genesis of bursting oscillations in the HindmarshRose model and homoclinicity to a chaotic saddle," Physica D, vol. 62, no. 1-4, pp. 263-274, 1993.

[56] X. L. Ding, Y. Y. Li, Q. H. Li, H. G. Gu, and W. Ren, "Bifurcation of periodic firing patterns in neuronal model," Journal of Dynamics and Control, vol. 7, no. 4, pp. 297-301, 2009 (Chinese).
[57] D. Linaro, A. Champneys, M. Desroches, and M. Storace, "Codimension-two homoclinic bifurcations underlying spike adding in the Hindmarsh-Rose burster," SIAM Journal on Applied Dynamical Systems, vol. 11, no. 3, pp. 939-962, 2012.

[58] L. Djouhri, S. Koutsikou, X. Fang, S. McMullan, and S. N. Lawson, "Spontaneous pain, both neuropathic and inflammatory, is related to frequency of spontaneous firing in intact C-fiber nociceptors," Journal of Neuroscience, vol. 26, no. 4, pp. 1281$1292,2006$. 


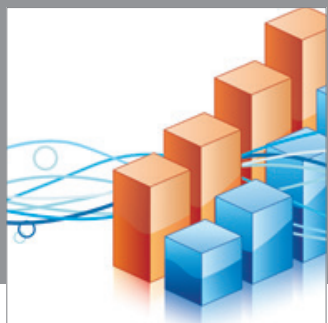

Advances in

Operations Research

mansans

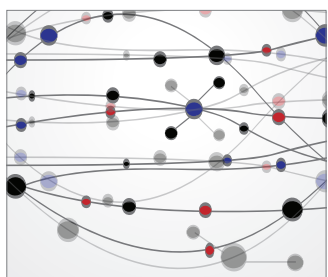

The Scientific World Journal
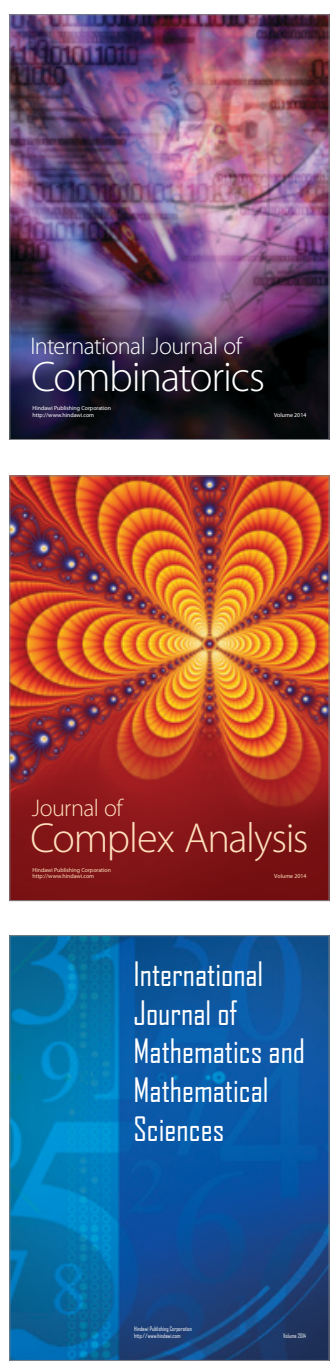
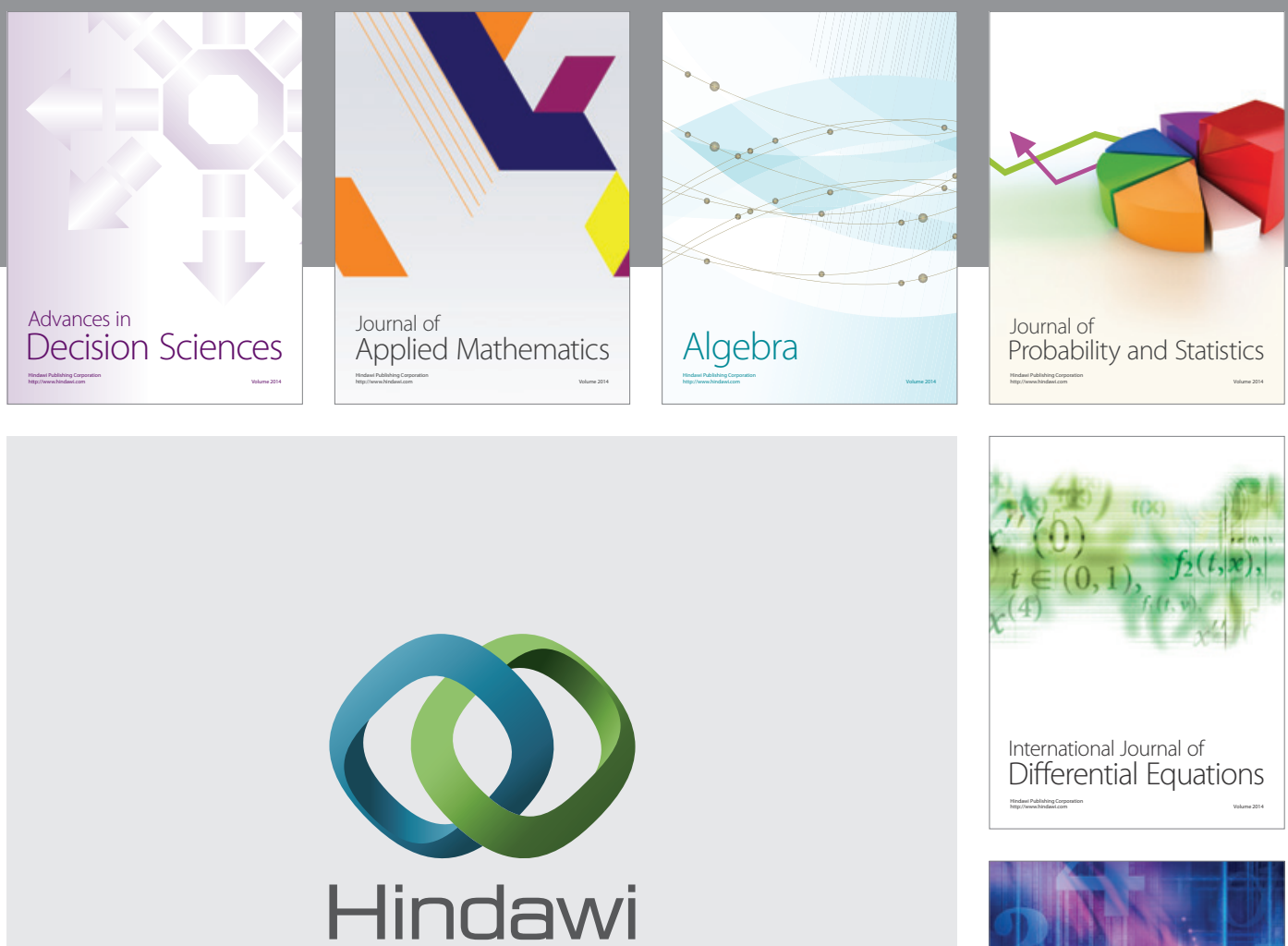

Submit your manuscripts at http://www.hindawi.com
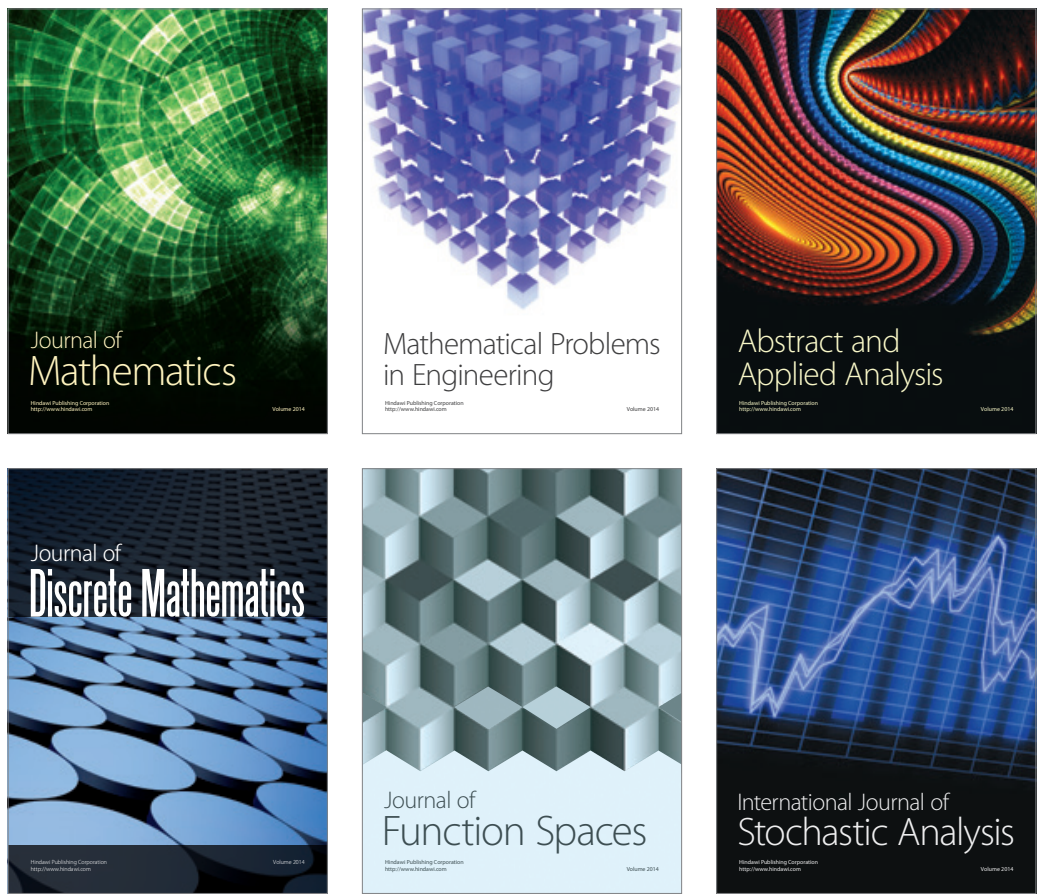

Journal of

Function Spaces

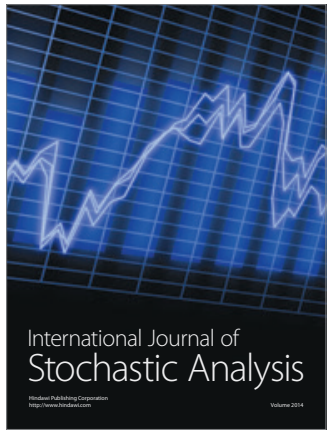

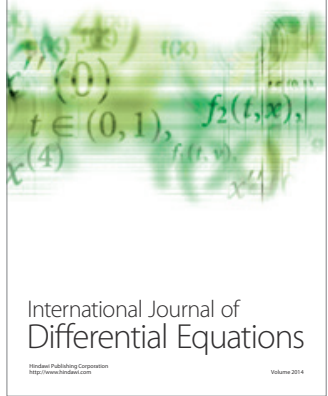
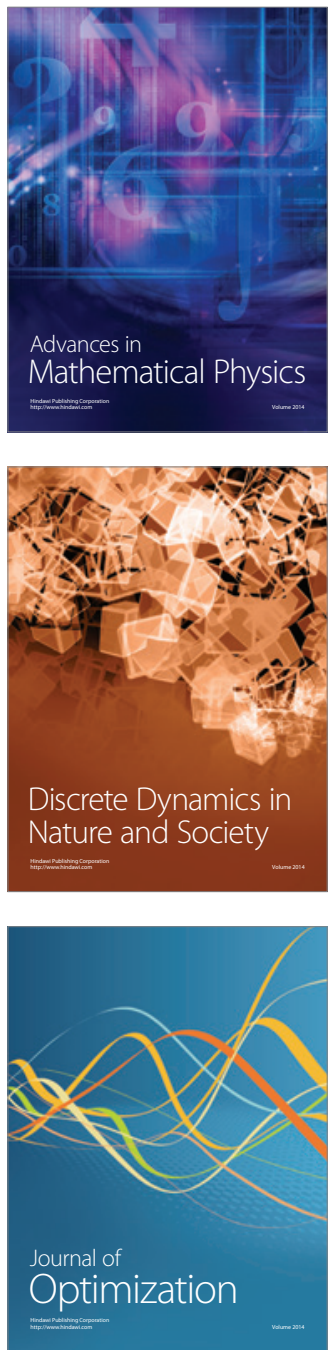